\title{
EL ESTRIBILLO EN LAS "COPLAS DEL ALMA QUE PENA POR VER A DIOS", DE SAN TUAN DE LA CRUZ
}

Para cualquier lector asiduo de San Juan de la Cruz, la riqueza y la hermosura de sus poemas son hechos indiscutibles. Sin duda alguna, San Juan representa una cúspide dentor de la poesía en lengua española. Esto podría deberse, entre otras cosas, a que el autor maneja la enorme complejidad del mensaje poético de tal modo que, al lector, el mensaje no le parece ni difícil ni oscuro.

Pero tomemos un ejemplo. En este trabajo intentaré llevar a cabo un análisis lo más científico y objetivo posible, para fundamentar juicios como los que se mencionan en el párrafo anterior, de manera que éstos cobren realmente sentido.

ALgunas geNeralidades SOBRE EL MENSAJE POÉTICO

1. Multiplicidad de códigos. No insistiré, en este momento, en que el mensaje poético presenta una complejidad que supera en mucho la de cualquier otro tipo de mensaje en la lengua natural. Esta complejidad resulta del hecho de que el texto poético se cifra en más de un código: en primer lugar en el de la lengua natural a que pertenece; en segundo lugar en el código característico de la lengua poética, que establece sus propias reglas y suspende algunas de las de la lengua natural. En tercer lugar podemos llamar código cultural a otro ámbito de invariantes, ideológicas, que determinan, por ejemplo, cuáles paradigmas del código poético han de actualizarse. ${ }^{1}$ En un texto como el que aquí nos

1 José Pascual Buxó está estudiando actualmente los sistemas o códigos que se actualizan en un texto poético, y llama "semiológico" al tercer código que se menciona aquí. También Juri M. Lotman, en Die Struktur 
interesa, estos tres códigos se entrecruzan. En general podemos añadir que los dos segundos son verbalizables por medio del primero, es decir, que pueden aparecer en un mensaje lingǘstico, pero no necesitan hacerlo. Al aludir al código ideológico no me refiero pues a la ideología (o particular reticulación del mundo) que cada lengua conlleva, sino precisamente a aquellos elementos ideológicos o culturales que pueden ser manifestados a través de diversos lenguajes (por ejemplo, el pictórico). Con esto quiero recalcar que este código no es exclusivo de los mensajes lingüísticos, y ni siquiera de los poéticos, aunque aparezca en ellos.

El texto poético, según nuestra hipótesis, hace coincidir en algún punto estos tres códigos. La lengua natural sirve de materia prima para actualizar elementos propios de los demás sistemas. Esto podría esquematizarse de la siguiente manera:

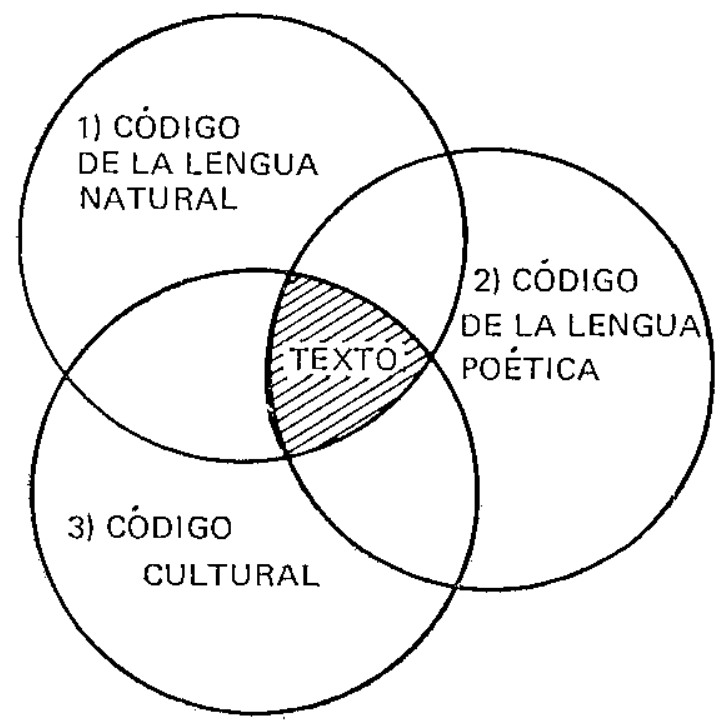

lilerarischer Texte, recalca la importancia de los elcmentos culturales, aunque no los define como parte de un código preciso, i.e., como sistema de invariantes culturales. 
Este cruce da cuenta, al menos de manera parcial, de la complejidad y de la enorme carga informativa que el mensaje poético posee. Sin embargo hay asimismo otro aspecto importante, que trataré a continuación, para la explicación. de estos fenómenos.

2. Semantización de niveles. Básicamente la finalidad esencial de todo mensaje es la transmisión de significados. El mensaje poético, dependiente de múltiples paradigmáticas, presenta una especial complejidad de significados. Conduce a ella una de las invariantes propias del código de la lengua poética, que consiste en la semantización de todos los niveles lingüísticamente analizables. Esto quiere decir que, en el mensaje poético, aquello que en cualquier otro tipo de mensaje lingüístico serviría únicamente como medio para la transmisión - a saber, los elementos fonológicos y morfosintácticos-- adquiere significación propia, lo cual aumenta de modo.considerable la carga informativa. Este hecho es, precisamente, la causa de que el contenido del mensaje poético sea inseparable de su forma, puesto que todo en él reviste una dimensión semántica, como trataré de comprobar más adelante.

3. El método de análisis. Como resultado de lo expuesto hasta aquí, proponemos un análisis que intente dar cuenta de los miembros paradigmáticos relevantes de cada uno de los códigos que el texto actualice, y esto en los diversos niveles analizables lingüisticamente. Creo recomendable proceder por niveles, puesto que resulta más claro y sistemático. Pero no hay que olvidar en ningún momento que el texto funciona como totalidad, y como tal transmite sus significados complejos. Además todo contribuye al aspecto semántico del texto, que es el más importante; los ejes de significación que lo cruzan en los diversos niveles, actúan siempré entrelazados y en relación con otros ejes u otros niveles. El aspecto semántico cubre, pues, cada una de las subestructuras textuales. Por lo mismo distingo los niveles siguientes:

a) Nivel fonosemạntico. 
b) Nivel morfosintáctico-semántico.

c) Nivel léxico-semántico.

Esta distinción no concuerda con la hecha tradicionalmente, ya que recalca en todos los niveles un aspecto semántico especial. Tal vez sea importante aclarar que, en el tercer nivel, no se entiende como "semántico" lo mismo que sería analizable en un mensaje no poético, es decir, los denotata de la lengua referencial, en la cual el significado y el significante de los signos son solidarios y complementarios. La distinción anterior se refiere, en los tres niveles, justamente a esa semantización específica de la lengua poética, a la cual me referí en el segundo inciso. Al recalcar el aspecto semántico en cada uno de los niveles, es importante subrayar que cualquier característica, presente en alguno de ellos, tiene repercusiones semánticas. Así, es inseparable el aspecto fónico del semántico, por ejemplo, la carga significativa de una aliteración, del significado de la o las palabras en que esta aliteración aparece. Asimismo estoy totalmente de acuerdo con Hjelmslev cuando se opone a separar la morfosintaxis de la semántica, ${ }^{2}$ porque, en efecto, funcionan como totalidad. Pero a pesar de ello considero útil para el análisis hacer una distinción de niveles por razones de método, lo cual ni contradice que cada uno de estos niveles funcione como uno de los aspectos de la expresión de los contenidos, ni que todos estos niveles se den a un mismo tiempo y, por ser simultáneos, son separables solamente en un análisis objetivo y no en el momento de la aprehensión del mensaje.

\section{II}

EL TEXTo

Analizaré aquí un texto de San Juan de la Cruz para tratar de comprobar algunas hipótesis que presentaré a conti-

2 CE. Louis Hjelmslev, Prolegómenos a una teoria del lenguaje, Gredos, Madrid, 1971. 
nuación, y que se refieren tanto a la complejidad y riqueza del mensaje poético en general, como al caso especial del estribillo de este poema. He aquí el texto: ${ }^{3}$

COPLAS DEL ALMA QUE PENA POR VER A DIOS

Vivo sin vivir en $\mathrm{mi}$

y de tal manera espero,

que muero porque no muero.

I. En mí yo no vivo ya, y $\sin$ Dios vivir no puedo; pues sin $\mathrm{kl}$ y $\sin$ mí quedo, este vivir ¿qué será?

Mil muertes se me hará, pues mi misma vida espero, muriendo porque no muero.

II. Esta vida que yo vivo es privación de vivir; y así, es continuo morir hasta que viva contigo. Oye, mi Dios, lo que digo, que esta vida no la quiero; que muero porque no muero.

III. Estando ausente de $\mathrm{Ti}$, ¿qué vida puedo tener, sino muerte padescer, la mayor que nunca vi? Lástima tengo de mi, pues de suerte persevero, que muero porque no muero.

IV. El pez que del agua sale aun de alivio no caresce, que la muerte que padesce, al fin la muerte le vale. ¿Qué muerte habrá que se iguale

3 La versión que manejo procede de: San Juan de la Cruz, Vida y obras, $5^{\text {a }}$ ed., Madrid, B. A. C., 1964, pp. 929-930. 
a mi vivir lastimero,

pues, si más vivo, más muero?

V. Cuando me pienso aliviar de verte en el Sacramento, háceme más sentimiento

el no te poder gozar; todo es para más penar, por no verte como quiero, y muero porque no muero.

VI. Y si me gozo, Señor, con esperanza de verte, en ver que puedo perderte, se me dobla mi dolor; viviendo en tanto pavor y esperando como espero, muérome porque no muero.

VII. Sácame de aquesta muerte, mi Dios, y dame la vida; no me tengas impedida en este lazo tan fuerte; mira que peno por verte, y mi mal es tan entero, que muero porque no muero.

VIII. Lloraré mi muerte ya y lamentaré mi vida en tanto que detenida por mis pecados está. ¡Oh mi Dios!, ¿cuándo será cuando yo diga de vero: vivo ya porque no muero?

Se trata de un poema estrófico popular que presenta un tipo de glosa, es decir, un estribillo y una serie de mudanzas que repiten, al final, parte del estribillo, a veces con ligeras variaciones. El estribillo consta de un terceto con rimas abb. Las estrofas de siete versos contienen una redondilla de rimas abrazadas, cddc, y un terceto, cbb, que remite, 
tanto por rima como por ritmo, al estribillo, pero que igualmente se enlaza con la estrofa.

Las estrofas constan de versos octosílabos polirrítmicos en combinaciones diversas. El estribillo presenta una mayor regularidad en cuanto a la combinación de sus octosílabos. En la mayoría de las apariciones (presentación y estrofas I, II, III, V, VI y VII) el segundo verso es un octosílabo trocaico con acentos a partir de la tercera sílaba (oo óo óo óo), y el tercer verso (con excepción de la estrofa VI) un octosílabo mixto con anacrusis, pero con un acento obstructivo de ritmo sobre la sexta sílaba (o óo óoó óo). En las variaciones ritmicas que se dan en la estrofa IV, tercer verso de la VI y en la estrofa VIMI, se trata siempre de octosílabos dactílicos (óoo óoo óo).

III

\section{El estribillo}

El hecho de la repetición de un estribillo o refrán constituye un problema interesante para el análisis poético. ${ }^{4}$ En un texto poético una repetición difiere totalmente de cualquier repetición que se dé en un texto no poético. En éste una repetición es redundante y remite cada vez al mismo sentido. En el texto poético, al contrario, las repeticiones no son nunca idénticas ni redundantes $\%$, por la pertenencia del texto a varias paradigmáticas, se produce la polisemia que lo caracteriza y que hace que cada repetición se cargue con matices de sentido diferentes.

En nuestro caso concreto la vuelta del estribillo tiene varias funciones. Por una parte al repetirse de manera casi idéntica (en cuanto a rima, ritmo y sentido) en las tres primeras estrofas, ayuda a establecer el sistema ideológico de partida que priva en la primera mitad del poema: una vida (terrena) despreciable, una situación de desesperanza. Por

* Este problema ha sido estudiado por Carlos Bousoño, Teoria de la expresión poética, Gredos, Madrid, 1966. 
otra parte al introducir variaciones (IV, vi, vIII) provoca una tensión en ese sistema ya establecido, y lo modifica, reforzando el cambio de actitud y de enfoque (Dios ya está cercano, y no distante como en la primera mitad del poema) que se da en las mudanzas: hay un viraje hacia la esperanza desde que hay, en esta vida terrena, ocasión de vislumbrar y anhelar la vida eterna y verdadera. La variación fundamental se encuentra al final del poema, en donde se resuelve la paradoja presentada al principio y, por tanto, se llega a la situación opuesta a la inicial. Dentro de la paradigmática cultural esta solución remite a la clave en la significación total del poema: la salvación del alma.

Todo lo anterior, naturalmente, ha sido dicho en forma sucinta, y solamente un análisis exhaustivo del poema completo nos llevaría a verio con claridad.

No hay que olvidarnos tampoco de que, por constituir el principio de nuestro texto, el estribillo le da al lector las claves necesarias para su inserción en los códigos que actualiza. En otras palabras el principio del poema, que en este caso es precisamente la presentación del refrán, señala al texto como un mensaje poético, con sus características peculiares (multiplicidad de códigos, semantización de niveles). En este análisis trataré de demostrar la existencia de estas características en la primera presentación del estribillo.

Por otra parte el estribillo encierra ya, y en su mayor concentración, el problema semántico-ideológico que va·a desarrollar el poema en cada estrofa, en sus diferentes aspectos. Por ello el refrán constituye el mejor ejemplo para una muestra de análisis del mensaje poético, puesto que presenta sus características en su mayor complejidad.

\section{IV}

ANÁLISIS DE LA PRESENTACIÓN DEL ESTRIBILLO

Intentaré aquí proceder por niveles, de acuerdo con lo expuesto en la primera sección de este trabajo. Aclaro, sin embargo, que atenderé a las interrelaciones de un nivel con 
otro, de modo que se hace difícil discernir, por ejemplo, si se trata más bien de un fenómeno perteneciente al nivel morfosintáctico o léxico-semántico. En casos como ese, trataré el problema al hablar del nivel en el cual se hayan dado las descripciones, para su mejor comprensión.

a) Nivel fonosemántico. Antes que nada cabe señalar que cualquier característica del significante tiene una correlación con el significado. Así, dos palabras con significantes similares, por ejemplo desde el punto de vista fónico, producen, dentro del código de la lengua poética, asociaciones semánticas que pueden no existir en textos no poéticos. ${ }^{5}$ Este es uno de los medios que se utilizan para crear un sistema propio de sinonimia y antinomia.

En el estribillo que nos ocupa notamos en el primer verso una aliteración de $i$ que se da en todas las sílabas tónicas:

\section{Vivo $\sin$ vivir en $\mathrm{m} i$}

A partir de la palabra/sin/ todas las vocales son palatales, de modo que podemos ver ahí una escisión en el verso. Esto subrayaría el sema de "carencia" que esta palabra confiere al lexema que modifica. Además ésta relaciona, a manera de eje, el mismo verbo/vivir/ utilizado una vez en primera persona y la otra en infinitivo. Por esta aparente homogeneidad contextual en la que se sitúa esta preposición $/ \sin /$, se refuerza también su significado que sobresale como elemento no homogéneo. Asimismo la aliteración relaciona $/ \sin /$ con /mí/. Todas las palabras relacionadas ponen de manifiesto, en su relación, tanto sus semejanzas como sus diferencias. Se dan así, en el primer verso, los siguientes pares debidos a la aliteración y a los acentos:

$$
\begin{aligned}
& \text { vivo : } \sin \text { vivo: vivir } \\
& \sin : \text { vivir } \sin : \mathrm{m}^{\prime}
\end{aligned}
$$

" CE. Jakobson, "Lingüística y poćtica", en Ensayos de lingüistica general, Seix Barral, Barcelona, 1975, y también Lotman, op. cit. 
Además se asemejan las preposiciones / sin/: /en/, tanto por la función como por las aliteraciones (palatal, nasal).

El segundo verso,

$$
\text { y de tal manera espero, }
$$

presenta sus crestas acentuales sobre la vocal media $a$ y la palatal $e$. Las relaciones que se producen son:

$$
\text { tal : manera manera : espero }
$$

En este último par la paronomasia -era : -ero realza las diferencias entre las categorías y, a la vez, se "contaminan" una a la otra: /tal manera/, en relación con /espero/, dota de 'intensidad' al verbo, mientras que /espero/ verbaliza, si se puede decir así, a / tal manera/.

Por otra parte/tal/ y /espero/ introducen las primeras oclusivas sordas, lo que las asemeja también.

El tercer verso,

$$
\text { que muero porque no muero, }
$$

presenta una tendencia clara hacia las vocales velares: $u e$, $o$. Dos de los acentos principales recaen sobre la misma palabra, recalcando así, en un significante homófono, diferencias de sentido, ya que el mismo hecho de la repetición hace que cambie de significado en el código poético. Además el acento obstructivo de ritmo sobre /no/ refuerza la negación $y$, con ello, la paradoja.

El acento secundario en la cuarta sílaba, por-, es reforzado asimismo por la repetición de /que/, Nuevamente un contexto fónico homólogo hace resaltar el elemento distintivo, esta vez, en cuanto a su sema de 'causalidad' : que : porque.

En sentido vertical hay una oposición fonológica entre el primero y el tercer versos, debida a las vocales palatales en el primero y a las velares en el tercero. Por otra parte las nasales en los tres versos relacionan / 1 i : /manera/; 
/muero/ : /no muero/. Los campos semánticos de todos estos signos son importantes para el poema.

La rima entre / espero/: / no muero/ recalca la sinonimia que el texto establece entre estas dos unidades. (Cf. más adelante.)

b) Nivel morfosintático semántico. El estribillo presenta un periodo con tres oraciones coordinadas - al menos formalmente-, la tercera de las cuales presenta, a su vez, una subordinada causal. La primera coordinación, introducicla por la conjunción copulativa / / / es, sin embargo, más bien una adversación, tanto por el sentido como por el hecho formal de que su estructura es inversa a la de la primera oración principal:

1) verbo - complementos circunstanciales.

2) complemento circunstancial - verbo.

De este modo, aunque por una parte los dos primeros versos son reiterativos en cuanto a significación (cf. infra), por la otra se oponen. La posición de los verbos, sinónimos aquí, /vivo/y /espero/, destruye y refuerza al mismo tiempo su coincidencia semántica, provocando tensión.

Todos los verbos que aparecen en modo personal están en primera persona y ocupan posiciones acentuadas en el verso:

Viचo sin vivir en mí

y de tal manera espero, que muero porque no muero.

La posición produce las equivalencias en todos los sentidos:

$\begin{array}{rlll}\text { vivo } & : \text { muero; } & \text { vivo } & \text { no muero; } \\ \text { espero } & \text { no muero; } & \text { vivo } & \text { espero; }\end{array}$

muero : espero.

La columna de la izquierda muestra las oposiciones provo- 
cadas por los verbos que ocupan una posición homóloga; la de la derecha las oposiciones resultantes de una posición contraria. Al mismo tiempo las posiciones homólogas están ocupadas por verbos que se oponen en los paradigmas de la lengua natural y que, a la vez, revisten una carga significativa importante dentro de los paradigmas culturales. La segunda columna contiene, en las oposiciones que en el poema se dan de arriba hacia abajo, sinónimos, el primer par, de la lengua natural, el segundo, de una sistema ideológico. En la pareja de en medio, que contiene los verbos que se oponen de abajo hacia arriba, hay una relación antinómica ya sólo a nivel ideológico (muero : espero), en donde el significado de /espero/ ya ha sido establecido antes.

Las dos paradojas que se presentan en el primero y en el tercer versos, tienen diferente fuerza, cuya señal es una marca morfológica distinta. La segunda paradoja/muero porque no muero/ provoca mayor ruptura que la primera / vivo sin vivir/, puesto que en las formas verbales coincide la persona gramatical.

Vimos ya, al hablar del nivel fonosemántico, que el sema de 'causalidad' que porta el nexo de la oración subordinada es reforzado por el contorno fonológico. Esto, unido al hecho de que sea un término homófono, y de que el primer término de la paradoja sea justamente la causa del otro, que aparentemente lo niega, otorga al recurso su mayor fuerza.

De todo esto podemos deducir que las semejanzas y oposiciones morfosintácticas provocan determinados juegos semánticos. Esto nos comprueba la semantización de este nivel.

c) Nivel léxico-semántico. La característica principal, fácil de advertir a simple vista, es el juego de homónimos y antónimos que presenta este estribillo. Llamo homónimos a las repeticiones, puesto que ya mencionamos con anterioridad que en un texto poético éstas no son posibles en la misma medida en que lo son en un texto diverso, en lengua natural. Aquí, ya que cualquier característica en alguno de los niveles se correlaciona con el aspecto semántico, las posiciones y funciones diferentes provocan, naturalmente, un cambio en el significado. Así, no es lo mismo 
vivo (verbo ppal.) : sin vivir (término de la prep., compl. circunstancial)

muero (verbo orac. ppal.) : no muero (vbo. orac. subord.)

El segundo verso no presenta repeticiones verbales homónimas. Sin embargo el juego existente entre/manera/: / espero/ carga al verbo de una intensidad especial (Cf. antes.)

En el eje vertical del estribillo, la oposición léxica fundamental es la de los antónimos de la lengua natural

$$
\text { 'vivir' : 'morir', }
$$

que forman, a la vez, uno de los pares básicos en cualquier sistema ideológico.

El eje horizontal presenta, en los juegos de estos dos antónimos, la doble paradoja:

\section{vivo $(+)$ vs. $\sin$ vivir $(\sqcup)$ \\ muero $(-)$ vs. no muero $(t)$}

En ambos casos el segundo elemento es antecedido por un signo de negación, pero el efecto semántico es opuesto. Los signos entre paréntesis indican la carga semántica positiva para 'vivir', es decir, 'no morir', 'ser'; y negativa para 'morir', es decir, 'no vivir', 'no ser'. Se produce así un efecto quiasmático:

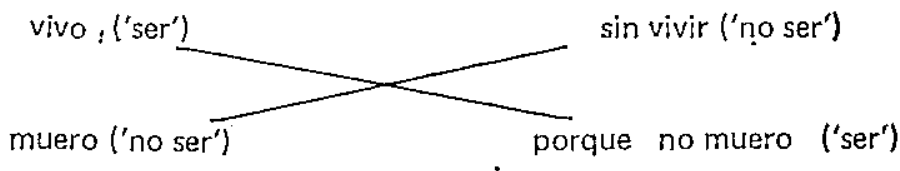

La situación inicial y la final son idénticas, o sea, de desesperación, puesto que se trata de un vivir que se contradice. El hecho de ser posible una tal contradicción, utilizando los vocablos de la lengua natural, nos remite inmediatamente a otro código, el poético, que restituye la logicidad de una 
predicación como ésta y, asimismo, a un determinado sistema cultural o ideológico que dote de sentido - de un sentido determinado- a la misma. Son estos dos códigos, superpuestos al de la lengua natural, los que pueden explicarnos la posibilidad de la predicación del tercer verso en el cual, a nivel de lengua denotativa, la causa contradice lo expresado en la oración principal.

El verbo/espero/, tanto por la rima como por la homologación posicional con /no muero/, e igualmente por la conjunción copulativa que es el nexo de la segunda oración, se asemeja y acerca a los semas presentes en 'vivo', que es sinónimo como ya vimos de 'no muero'. Hay de este modo una relación sinonímica en este texto concreto, entre 'vivo', 'espero' y 'no muero', que adquiere características temporal y positiva, frente a 'muero', intemporal y negativo. Este hecho, el hacer coincidir la vida con una espera (de la muerte), nos remite con toda claridad a un código ideológico que explica el tipo de espera, precisanente, y cuyos paradigmas son comprobables también en otra clase de textos (filosóficos y teológicos, por ejemplo).

De esta manera el estribillo del poema de San Juan presenta una problemática ideológica compleja. En ésta, la vida (en su acepción inmediata) no es tal, no conlleva los rasgos positivos propios de la lengua natural. En este esperar (la muerte) que es la vida, los rasgos negativos atribuidos a 'morir' en la lengua natural son los que, en realidad, corresponden a esta vida. Sólo la muerte (en su acepción cultural, es decir, cristiana) traerá consigo la vida verdadera (en esta misma acepción). ¿Cómo es posible esta interpretación? Como hemos visto, la introducción del código poético hace que se suspendan las oposiciones propias del código de la lengua natural. Precisamente mediante las predicaciones paradójicas, de acuerdo con los recursos del segundo código, puede llegarse a las significaciones del tercero. Dicho de otro modo: al decir

$$
\text { /vivo sin vivir en mil }
$$

se ha introducido un nuevo código que hace que esta predi- 
cación, aparentemente ilógica, sea posible y significativa. Pero el significado que le es propio hay que aprehenderlo de un tercer sistema, ideológico o cultural, que explica en qué sentido preciso 'vivo sin vivir en mí. El texto nos dota, él mismo, de las claves: en las mudanzas que glosan este estribillo se establece perfectamente la inclusión de este texto en un contexto cultural determinado $(/ y \sin$ Dios vivir no puedo...//).

Asi, los significados propios de las palabras más importantes dentro del estribillo, las parejas opositivas entre los antónimos naturales, resultan ser precisamente los contrarios a los significados de la lengua denotativa o de un texto no poético. Podemos entenderlo así: Como 'vivo sin vivir en mí, el primer / vivo/ alude, en realidad, a un 'muero', puesto que es/sin vivir/, lo que se interpreta como 'en ausencia' (de Dios), esto es, sólo en cuanto a la vida corporal. El segundo/vivir/, en cambio, se refiere a la vida del alma. Así, este 'vivir sin vivir' conlleva, en total, una carga negativa. El segundo verso, sin embargo, carga positivamente al poema, pues, al 'esperar' (la vida eterna), se desea la vida del alma. Pero en todas las estrofas, con excepción de la última, el tercer verso del estribillo retorna a la situación inicial con la otra paradoja: /muero por que no muero/. El primer /muero/ alude a la vida anímica, la vida verdadera, y se interpreta como 'sufro'; el segundo, en cambio, se refiere a la vida terrena, corporal, que es detestable dentro de la paradigmática de este código cultural.

Resulta interesante trazar unos esquemas para ilustrar esto con mayor claridad. El lector, al enfrentarse a este texto, no desconoce los significados que los lexemas antonímicos tienen en la lengua natural, pero los suspende $y$, por las correlaciones en todos los niveles, atribuye a estas palabras nuevos significados. Sin embargo los que tienen normalmente no dejan de actuar, están ahí al mismo tiempo, provocando tensión cuando no coinciden. Así, si representamos la contraposición entre la vida del cuerpo y la del alma, y los valores positivo o negativo, con respecto a éstas, obtenemos el siguiente resultado: 
1)

\begin{tabular}{|l|}
\hline 'vivo sin vivir en mi' \\
\hline + cuerpo - alma \\
\hline
\end{tabular}

2)

\begin{tabular}{|c|}
\hline$y$ de tal manera espero'' \\
\hline+ aima \\
\hline
\end{tabular}

3)

\begin{tabular}{|c|}
\hline que muero porque no muero' \\
\hline$\rightarrow$ alma + cuerpo \\
\hline
\end{tabular}

Resultado de la predicación:

- valor ideológico

+ valor ideológico

- valor ideológico

En el desarrollo del poema las estrofas establecen primero, y matizan luego, esta problemática, y van resolviendo la enorme tensión producida tanto por el juego antonímico $y$ sinonímico a la vez, como también por la alternancia $(-/+/-)$ en los tres versos en cuanto a la carga ideológica. Además las estrofas, como ya indiqué anteriormente, insertan con toda claridad el texto dentro de la paradigmática ideológica de la mística: la muerte (corporal) llevará a la vida verdadera (del alma), porque conducirá el alma hacia Dios. De igual manera hay otras muchas señales textuales que nos hacen introducir paradigmas culturales: desde la forma métrica, que remite a la poesía popular española, hasta los juegos complejos de oposiciones antonímicas y las paradojas, que remiten a la poesía trovadoresca de los cancioneros, y al barroco.

Las variantes principales del estribillo, en la estrofa iv y en la viII, son importantes para la interpretación de las significaciones totales. Desde el punto de vista formal, además de variar el ritmo, se oponen al resto de las vueltas en cuanto, mientras éstas siempre presentan en el segundo verso del terceto el lexema 'vida' o alguno de sus sinónimos, y en el tercero el lexema de 'muerte', en éstas dos estrofas el último verso presenta ambos términos opositivos, lo cual acentúa el problema presentado. En cuanto al valor ideológico, la cuatra estrofa lo conserva $(-/+/-)$; únicamente invierte, en el último verso, el orden: 


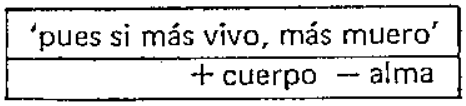

En cambio la resolución final anula, simultáneamente en los tres códigos, la paradoja, inexistente ya en

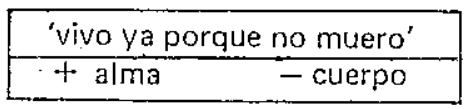

y esto cambia los valores ideológicos, dando la serie $-/+/+$, es decir, una predicación en donde los dos versos del estribillo tienen sólo marcas semánticas positivas. Ésta es la esperanza del cristiano, encontrar la vida verdadera de Dios. El hecho de que esta predicación sea formalmente paralela a la inicial (/muero porque no muero/), y que contenga verbos en presente de indicativo, recalca, a nivel ideológico, sus semejanzas (de posibilidad real) y diferencias (las situaciones opuesta).

Con un análisis de este tipo no se trata de demostrar significados que sean inasequibles para un lector competente. Al contrario, quien esté avezado en esta clase de textos, puede llegar perfectamente a aprehender sus significados. El análisis, empero, los comprueba objetivamente y los dota de mayor intensidad, puesto que nos hace ver de qué manera y hasta qué punto la intención semántica recorre todos los niveles del mensaje. Se producen asi significados nuevos, distintos a los de la lengua natural, enriquecidos por sus correlaciones mutuas en el texto e inseparables de ellas. De este modo se generan esos significados poéticos que, si bien son inexpresables en lengua denotativa, pueden describirse, con un metalenguaje, mediante un análisis por niveles, que muestre su complejidad y su intrincada interrelación estructural.

Margaruta Pease

Seminario de Poética.

Instituto de Investigaciones Filológicas. 